\title{
Algorithm for the Numerical Solutions of Volterra Population Growth Model with Fractional Order via Haar Wavelet
}

\author{
Rohul Amin ${ }^{1 *}$, Şuayip Yüzbaşı ${ }^{2}$, Liping Gao ${ }^{3}$, Muhammad Asif ${ }^{1}, \operatorname{Imran}_{K^{\prime}}{ }^{1}$ \\ ${ }^{1}$ Department of Mathematics, University of Peshawar, Pakistan \\ ${ }^{2}$ Department of Mathematics, Faculty of Science, Akdeniz University, Tr-07058, Antalya, Turkey \\ ${ }^{3}$ Department of Computational Mathematics, School of Sciences, China University of Petroleum, Qingdao, 266555, China \\ Email: raminmath@uop.edu.pk, suayipyuzbasi@gmail.com
}

\begin{abstract}
In this paper, a new collocation method based on the Haar wavelet is developed for the numerical solution of the Fractional Volterra Model (FVM) for the population growth of a species in a closed system. In the proposed method the derivative involved in the nonlinear model is approximated using Haar wavelet and the approximate expressions for the unknown function are obtained by the process of integration, the fractional derivative will be considered in the Caputo sense. The technique of residual correction, which aims to reduce the error of the approximate solution by estimating this error, is discussed in some detail. To show the computational efficiency of the proposed method, the residual correction technique is illustrated with an example. The numerical results show that the method is simply applicable, accurate, efficient, and robust.
\end{abstract}

Keywords: population dynamics, Fractional Volterras population model, fractional derivative, Nonlinear integro-differential equations, Haar wavelet, collocation method

\section{Introduction}

Fractional calculus is a 300 years old mathematical discipline. The concept to differentiate a fractional order was denied by Remain and Liouville. There exist numerous definition for fractional derivatives of order $\beta>0$ in the literature, for example, Remainn-Liouvlle fractional derivative (FD), Caputo FD, and so on. Remainn-Liouvlle FD of order $\alpha>0$ is not an integer as:

$$
\left(D^{\beta} f\right)(t)=\left(\frac{d}{d t}\right)^{n}\left(I^{n-\beta} f\right)(t), n-1<\beta \leqslant n,
$$

when $n \in \mathbb{Z}$. There are some disadvantages of this model the real-world phenomenon having FDEs. Because of this reason Caputo proposed a modified differential operator $\mathrm{D}^{\beta}$ of fractional order. The Caputo definition for the $\mathrm{FD}$ of order $\beta>0$ is:

$$
\left(D^{\beta} f\right)(t)=\frac{1}{\Gamma(n-\beta)} \int_{0}^{t}(t-\tau)^{n-\beta-1} f^{n}(\tau) d \tau, n-1<\beta \leq n
$$

where $t>0$ and $n$ is an integer. To tackle numerical problems in material science and engineering FDEs are numerous in modeling many phenomenon, however, there are most of the FDEs which do not have analytical solutions. The aim of this proposed research work is to find out an efficient and accurate numerical scheme by utilizing Haar wavelet collocation method (HWCM). HW depends on the functions which were presented by Alfred Haar in 1910. The HW functions are comprised of piecewise constant functions. HW belongs to the family of square functions (i.e. it might secure just the values $0,+1,-1)$. Such wavelets are numerically the most simple when compared with other wavelet families. HW is mathematically simple and integrated analytically arbitrary time, therefore it has been used in different approximation problems including integral and integro DEs. The Haar matrices contain many zeros, this influences the Haar transform faster than for other wavelet functions ${ }^{[1,2]}$. HWCM utilizes basic box functions, subsequently the formulation of numerical technique in light of HW is direct and includes lesser difficult work. Because of better approximating properties of HWCM 
gives more precise solutions as compared to other techniques. This technique is extremely advantageous for solving boundary value problems since the boundary conditions are taken into account automatically. HWCM is especially appropriate for systems involving abruptly varying functions. The HWCM is highly feasible for damage detection. By reason of these above highlights, we can attest that when compared with the established techniques, the HWCM is significantly richer in theory, more convenient in numerical calculations and, most of all, it is considerably quicker in information preparing. The main advantages of this method are its simplicity and less computation costs: it is due to the sparsity of the transform matrices and to the small number of significant wavelet coefficients. In comparison with existing numerical schemes to solve the FIDE, the Haar wavelet method is an improvement over other methods in terms of accuracy. Raza and $\mathrm{Khan}^{[3]}$ used the Haar wavelet series for the solution of neutral delay differential equations. Also, these authors utilized the Haar wavelet collocation method for the solution of higher-order two-point boundary value problems ${ }^{[4]}$. Islam et al.$^{[5]}$ found the numerical solution of second-order boundary-value problems by collocation method with the Haar wavelets.

In this paper, the FD will be discussed in the Caputo sense and is given by[6, 7]:

$$
D^{\beta} f(x)=\frac{1}{\Gamma(n-\beta)} \int_{0}^{x} \frac{f^{(n)}(t) d t}{(x-t)^{1+\beta-n}}, \beta>0,
$$

if: $n-1<\beta<n, n$ is a positive integer, $x>0$ : Caputo FD operator is a linear operator

$$
D^{\beta}(\lambda f(x)+\mu g(x))=\lambda D^{\beta} f(x)+\mu D^{\beta} g(x),
$$

where $\mu$ and $\lambda$ are constants.

Also we have $[8,9]$ :

$$
D^{\beta} C=0 \text {, if } \mathrm{C} \text { is constant, }
$$

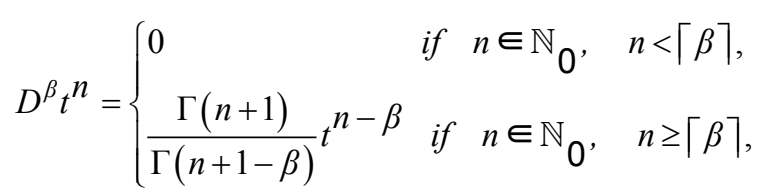

where $\lceil\beta\rceil$ denotes the ceiling function and $\mathbb{N}_{0}=\{0,1,2,3 \ldots\}$. For detail on Caputos derivative see $[10,11]$, delay DEs see $[12$, $13]$ and fractional delay DEs see [14-16]

In this paper, we consider the FVM of growth species $[17,18]$. The model is nonlinear FV integro DE

$$
\frac{d^{\beta} u(t)}{d t^{\beta}}=a u^{2}(t)-b u^{2}(t)-c u^{2}(t) \int_{0}^{t} u(x) d x, t \geq 0, x \leq R<\infty,
$$

with initial condition

$$
u(0)=\lambda
$$

where $\beta$ is the order of the FD and $0<\beta<1$, therefore $n=1, R$ is a constant, $a>0$ is the birth rate, $b>0$ is the crowding coefficient, and $c>0$ is the toxicity coefficient, $u=u(t)$ is the population of identical individuals at time $t[19,20]$.

The paper is organized in the following structure. In Section 2, the definition of HW is given. The numerical method for the solution of the FV population growth model based on HWCM is developed in Section 3. The FD is described in the Caputo sense. In Section 5 numerical experiments are performed. At last, conclusions are drawn in Section 6.

\section{Haar wavelet}

The scaling and mother wavelet functions for the Haar family on $[0 ; 1)$ are [21]: 


$$
\begin{aligned}
& \mathbb{H}_{1}(x)= \begin{cases}1 & \text { for } x \in[0,1), \\
0 & \text { otherwise. }\end{cases} \\
& \mathbb{H}_{2}(x)= \begin{cases}1 & \text { for } x \in[0,0.5), \\
-1 & \text { for } x \in[0.5,1), \\
0 & \text { otherwise. }\end{cases}
\end{aligned}
$$

The other functions in wavelet family are obtained from the mother wavelet and given as:

$$
\mathbb{H}_{i}(x)=\left\{\begin{array}{c}
1 \text { for } x \in\left[\delta_{1}, \delta_{2}\right), \\
-1 \text { for } x \in\left[\delta_{2}, \delta_{3}\right), \\
0 \text { otherwise, }
\end{array}\right.
$$

where

$$
\delta_{1}=\frac{k}{m}, \quad \delta_{2}=\frac{k+0.5}{m}, \quad \delta_{3}=\frac{k+1}{m} .
$$

Here $m=2^{j}$ where $j=0,1,2, \ldots, J$, the rank $k$ is defined by $k=0,1,2, \ldots, m-1$. The connection between numbers $k$, $i$ and $m$ is $i=k+m+1$.

We present the following representation:

$$
p_{i},{ }_{1}(x)=\int_{0}^{x} \mathbb{H}_{i}(t) d t
$$

This integral can be calculated using Eq. (10) which yields:

$$
p_{i},{ }_{1}(x)=\left\{\begin{array}{cl}
x-\zeta_{1} & \text { for } x \in\left[\zeta_{1}, \zeta_{2}\right) \\
\zeta_{3}-x & \text { for } x \in\left[\zeta_{2}, \zeta_{3}\right) \\
0 & \text { elsewhere }
\end{array}\right.
$$

\section{Numerical method}

In this section, the proposed method is developed to obtain an approximation solution of Eq. (6) using HWCM. We follow the Haar wavelet method adopted by Lepik in [1]. Consider $\dot{u}(t)$ is square-integrable and hence it can be written as:

$$
\dot{u}(t)=\sum_{i=1}^{N} \lambda_{i} h_{i}(t)
$$

integrating we have

$$
u(t)=u_{0}+\sum_{i=1}^{N} \lambda_{i} p_{i},{ }_{1}(t)
$$

where $u_{0}=u(0)$.

By applying the Caputo derivative definition to Eq (6), we have 


$$
\frac{1}{\Gamma(n-\beta)} \int_{0}^{t}(t-\tau)^{n-\beta-1} u^{n}(\tau) d \tau=a t(t)-b u^{2}(t)-c u(t) \int_{0}^{t} u(x) d x
$$

Since $0<\beta<1$, therefore $n=1$, and we have

$$
\frac{1}{\Gamma(1-\beta)} \int_{0}^{t}(t-\tau)^{-\beta} \dot{u}(t)(\tau) d \tau=a t(t)-b u^{2}(t)-c u(t) \int_{0}^{t} u(x) d x .
$$

Now using Haar approximations we get

$$
\begin{aligned}
& \frac{1}{\Gamma(1-\beta)} \int_{0}^{t}(t-\tau)^{-\beta} \sum_{i=1}^{N} \lambda_{i} h_{i}(\tau) d \tau=a\left(u_{0}+\sum_{i=1}^{N} \lambda_{i} p_{i},{ }_{1}(t)\right)-b\left(u_{0}+\sum_{i=1}^{N} \lambda_{i} p_{i},{ }_{1}(t)\right)^{2} \\
& -c\left(u_{0}+\sum_{i=1}^{N} \lambda_{i} p_{i},{ }_{1}(t)\right) \int_{0}^{t}\left(u_{0}+\sum_{i=1}^{N} \lambda_{i} p_{i},{ }_{1}(x)\right) d x,
\end{aligned}
$$

The integrals are approximated using the following formula [5]

$$
\int_{a}^{b} f(t) d t \approx \frac{b-a}{N} \sum_{k=1}^{N} f\left(t_{k}\right)=\sum_{k=1}^{N} f\left(a+\frac{(b-a)(k-0.5)}{N}\right),
$$

so from above we have

$$
\begin{aligned}
& \frac{1}{\Gamma(1-\beta)} \frac{t}{N} \sum_{m=1}^{N}\left(t-\tau_{m}\right)^{-\beta} \sum_{i=1}^{N} \lambda_{i} h_{i}\left(\tau_{m}\right)=a\left(u_{0}+\sum_{i=1}^{N} \lambda_{i} p_{i},{ }_{1}(t)\right)-b\left(u_{0}+\sum_{i=1}^{N} \lambda_{i} p_{i},{ }_{1}(t)\right)^{2} \\
& -c\left(u_{0}+\sum_{i=1}^{N} \lambda_{i} p_{i},{ }_{1}(t)\right) \frac{t}{N} \sum_{m=1}^{N}\left(u_{0}+\sum_{i=1}^{N} \lambda_{i} p_{i},{ }_{1}\left(x_{m}\right)\right) .
\end{aligned}
$$

After simplification, we have

$$
\begin{aligned}
& \frac{1}{\Gamma(1-\beta)} \frac{t}{N} \sum_{m=1}^{N}\left(t-\tau_{m}\right)^{-\beta} \sum_{i=1}^{N} \lambda_{i} h_{i}\left(\tau_{m}\right)-a\left(u_{0}+\sum_{i=1}^{N} \lambda_{i} p_{i},{ }_{1}(t)\right) \\
& +b\left(u_{0}+\sum_{i=1}^{N} \lambda_{i} p_{i},{ }_{1}(t)\right)^{2}+c\left(u_{0}+\sum_{i=1}^{N} \lambda_{i} p_{i},{ }_{1}(t)\right) \frac{t}{N} \sum_{m=1}^{N}\left(u_{0}+\sum_{i=1}^{N} \lambda_{i} p_{i},{ }_{1}\left(x_{m}\right)\right)=0,
\end{aligned}
$$

putting the nodal points $\mathrm{t}_{j}, j=1,2,3, \ldots, N$, we obtain

$$
\begin{aligned}
& F_{j}=\frac{1}{\Gamma(1-\beta)} \frac{t_{j}}{N} \sum_{m=1}^{N}\left(t_{j}-\tau_{m}\right)^{-\beta} \sum_{i=1}^{N} \lambda_{i} h_{i}\left(\tau_{m}\right)-a\left(u_{0}+\sum_{i=1}^{N} \lambda_{i} p_{i},{ }_{1}\left(t_{j}\right)\right) \\
& +b\left(u_{0}+\sum_{i=1}^{N} \lambda_{i} p_{i},{ }_{1}\left(t_{j}\right)\right)^{2}+c\left(u_{0}+\sum_{i=1}^{N} \lambda_{i} p_{i},{ }_{1}\left(t_{j}\right)\right) \frac{t_{j}}{N} \sum_{m=1}^{N}\left(u_{0}+\sum_{i=1}^{N} \lambda_{i} p_{i},{ }_{1}\left(x_{m}\right)\right) .
\end{aligned}
$$




$$
j=1,2, \ldots, N
$$

This system is solved using Broyden's method. The Jacobian is

$$
J=\left[j_{j k}\right]_{N \times N},
$$

where

$$
\begin{aligned}
& J_{j k}=\frac{\partial F_{j}}{\partial \lambda_{k}}=\frac{1}{\Gamma(1-\beta)} \frac{t_{j}}{N} \sum_{m=1}^{N}\left(t_{j}-\tau_{m}\right)^{-\beta} h_{k}\left(\tau_{m}\right)-a p_{i},{ }_{1}\left(t_{j}\right)+2 b\left(u_{0}+i=\sum_{i=1}^{N} \lambda_{i} p_{i},{ }_{1}\left(t_{j}\right)\right) p_{k},{ }_{1}\left(t_{j}\right) \\
& +c\left(u_{0}+\sum_{i=1}^{N} \lambda_{i} p_{i},{ }_{1}\left(t_{j}\right)\right) \frac{t_{j}}{N} p_{k},{ }_{1}\left(x_{m}\right)+c p_{k},{ }_{1}\left(t_{j}\right) \frac{t_{j}}{N} \sum_{m=1}^{N}\left(u_{0}+\sum_{i=1}^{N} \lambda_{i} p_{i},{ }_{1}\left(x_{m}\right)\right)
\end{aligned}
$$

The solution of this system gives values of the unknown $\lambda_{i}, \mathrm{i}=1,2,3, \ldots, N$. The approximate solution at nodal point is calculated by putting $\lambda_{i}, i=1,2,3, \ldots, N$ in Eq. (14).

\section{Error estimation and residual correction}

In this section, we study the error estimation and residual correction of the FVM for the population growth model.

The residual function $R_{N}(t)$ as

$$
R_{N}(t)=\frac{d^{\beta} u_{N}(t)}{d t^{\beta}}-a u_{N}(t)+b u_{N}^{2}(t)+c u_{N}(t) \int_{0}^{t} u_{N}(x) d x
$$

Let us define error function as

$$
e_{N}(t)=u(t)-u_{N}(t)
$$

where $u(t)$ is exact solution. So

$$
u(t)=e_{N}(t)+u_{N}(t)
$$

also

$$
\dot{u}(t)-\dot{u}_{N}(t)=\left(u(t)-u_{N}(t)\right)^{\prime}=\left(e_{N}(t)\right)^{\prime}
$$

and

$$
\frac{d^{\beta} u(t)}{d t^{\beta}}-\frac{d^{\beta} u_{N}(t)}{d t^{\beta}}=\frac{d^{\beta}}{d t^{\beta}}\left(u(t)-u_{N}(t)\right)=\frac{d^{\beta}}{d t^{\beta}} e_{N}(t),
$$

subtracting Eq. (19) from Eq. (6), we have

$$
\frac{d^{\beta}}{d t^{\beta}}\left(u(t)-u_{N}(t)\right)=a\left(u(t)-u_{N}(t)\right)-b\left(u(t)-u_{N}(t)\right)^{2}-c\left(u(t)-u_{N}(t)\right) \int_{0}^{t}\left(u(x)-u_{N}(x)\right) d x-R_{N}(t)
$$

by using Eq. (21), Eq. (22) and Eq. (23), we have 


$$
\frac{d^{\beta}}{d t^{\beta}} e_{N}(t)=a e_{N}(t)-b\left(e_{N}^{2}(t)+2 e_{N}(t) u_{N}(t)\right)-c e_{N}(t) \int_{0}^{t} e_{N}(x) d x-R_{N}(t)
$$

after simplification, we have

$$
\frac{d^{\beta}}{d t^{\beta}} e_{N}(t)=\left(a-2 b u_{N}(t)\right) e_{N}(t) b e_{N}^{2}(t)-c e_{N}(t) \int_{0}^{t} e_{N}(x) d x-R_{N}(t)
$$

where $e_{N}(t)$ is unknown function. The initial condition for approximate solution $u_{N}(t)$ is

$$
u_{N}(0)=\lambda
$$

so initial condition for system (25) is

$$
e_{N}(0)=0
$$

By applying the Caputo derivative de nition to Eq (25), we have

$$
\frac{1}{\Gamma(n-\beta)} \int_{0}^{t}(t-\tau)^{n-\beta-1} e_{N}^{n}(t)(\tau) d \tau=\left(a-2 b u_{N}(t)\right) e_{N}(t)-b e_{N}^{2}(t)-c e_{N}(t) \int_{0}^{t} e_{N}(x) d x-R_{N}(t) .
$$

Since $0<\beta<1$, therefore $\mathrm{n}=1$, and

$$
\frac{1}{\Gamma(1-\beta)} \int_{0}^{t}(t-\tau)^{-\beta} e_{N}(t)^{\prime}(\tau) d \tau=\left(a-2 b u_{N}(t)\right) e_{N}(t)-b e_{N}^{2}(t)-c e_{N}(t) \int_{0}^{t} e_{N}(x) d x-R_{N}(t) .
$$

Let $\left[e_{N}, M(t)\right]$ be square integrable and hence it can be written as:

$$
\left[e_{N},{ }_{M}(t)\right]^{\prime}=\sum_{i=1}^{M} \zeta_{i} h_{i}(t)
$$

by applying integration, we obtain

$$
e_{N},{ }_{M}(t)=\sum_{i=1}^{M} \zeta_{i} p_{i},{ }_{1}(t)
$$

where $e_{N}(t)$ is approximated by $e_{N},{ }_{M}(t)$, in fact, $e_{N},{ }_{M}(t)$ is Haar error estimation for $e_{N}(t)$.

Applying the HW approximations, we have

$$
\begin{aligned}
& \frac{1}{\Gamma(1-\beta)} \int_{0}^{t}(t-\tau)^{-\beta} \sum_{i=1}^{M} \zeta_{i} h_{i}(\tau) d \tau=\left(a-2 b u_{N}(t)\right) \sum_{i=1}^{M} \zeta_{i} p_{i},{ }_{1}(t) \\
& -b\left(\sum_{i=1}^{M} \zeta_{i} p_{i},{ }_{1}(t)\right)^{2}-c \sum_{i=1}^{M} \zeta_{i} p_{i},{ }_{1}(t) \int_{0}^{t} \sum_{i=1}^{M} \zeta_{i} p_{i},{ }_{1}(x) d x-R_{N}(t),
\end{aligned}
$$

The integrals are approximated using the following formula [22] 


$$
\int_{a}^{b} g(t) d t \approx \frac{b-a}{M} \sum_{k=1}^{M} g\left(t_{k}\right)=\sum_{k=1}^{M} g\left(a+\frac{(b-a)(k-0.5)}{M}\right),
$$

so from above we have

$$
\begin{aligned}
& \frac{1}{\Gamma(1-\beta)} \frac{t}{M} \sum_{m=1}^{M}\left(t-\tau_{m}\right) \sum_{i=1}^{-\beta} \zeta_{i} h_{i}\left(\tau_{m}\right)=\left(a-2 b u_{N}(t)\right) \sum_{i=1}^{M} \zeta_{i} p_{i},{ }_{1}(t) \\
& -b\left(\sum_{i=1}^{M} \zeta_{i} p_{i},{ }_{1}(t)\right)^{2}-c \sum_{i=1}^{M} \zeta_{i} p_{i},{ }_{1}(t) \frac{t}{M} \sum_{m=1}^{N} \sum_{i=1}^{M} \zeta_{i} p_{i},{ }_{1}(x)-R_{N}(t) .
\end{aligned}
$$

After simplification, we get

$$
\begin{aligned}
& \frac{1}{\Gamma(1-\beta)} \frac{t}{M} \sum_{m=1}^{M}\left(t-\tau_{m}\right) \sum_{i=1}^{-\beta} \zeta_{i} h_{i}\left(\tau_{m}\right)-\left(a-2 b u_{N}(t)\right) \sum_{i=1}^{M} \zeta_{i} p_{i},{ }_{1}(t) \\
& +b\left(\sum_{i=1}^{M} \zeta_{i} p_{i},{ }_{1}(t)\right)^{2}+c \sum_{i=1}^{M} \zeta_{i} p_{i},{ }_{1}(t) \frac{t}{M} \sum_{m=1}^{M} \sum_{i=1}^{M} \zeta_{i} p_{i},{ }_{1}(x)-R_{N}(t)=0 . \\
& \text { Let } \quad F_{j}=\frac{1}{\Gamma(1-\beta)} \frac{t}{M} \sum_{m=1}^{M}\left(t-\tau_{m}\right) \sum_{i=1}^{-\beta} \zeta_{i} h_{i}\left(\tau_{m}\right)-\left(a-2 b u_{N}(t)\right) \sum_{i=1}^{M} \zeta_{i} p_{i},{ }_{1}(t) \\
& +b\left(\sum_{i=1}^{M} \zeta_{i} p_{i},{ }_{1}(t)\right)^{2}+c \sum_{i=1}^{M} \zeta_{i} p_{i},{ }_{1}(t) \frac{t}{M} \sum_{m=1}^{M} \sum_{i=1}^{M} \zeta_{i} p_{i},{ }_{1}(x)-R_{N}(t) .
\end{aligned}
$$

plugging the nodal points $t_{j}, j=1,2,3, \ldots, M$, we obtain the system of nonlinear equations

$$
\begin{aligned}
& F_{j}=\frac{1}{\Gamma(1-\beta)} \frac{t_{j}}{M} \sum_{m=1}^{M}\left(t_{j}-\tau_{m}\right)^{-\beta} \sum_{i=1}^{M} \zeta_{i} h_{i}\left(\tau_{m}\right)-\left(a-2 b u_{N}\left(t_{j}\right)\right) \sum_{i=1}^{M} \zeta_{i} p_{i}, 1\left(t_{j}\right)+b\left(\sum_{i=1}^{M} \zeta_{i} p_{i},{ }_{1}\left(t_{j}\right)\right)^{2} \\
& +c \sum_{i=1}^{M} \zeta_{i} p_{i},{ }_{1}\left(t_{j}\right) \frac{t_{j}}{M} \sum_{m=1}^{M} \sum_{i=1}^{M} \zeta_{i} p_{i},{ }_{1}(x)-R_{N}\left(t_{j}\right), \\
& j=1,2, \ldots, M .
\end{aligned}
$$

This system is solved by Broyden's method. Jacobian is

$$
\mathrm{J}=\left[\mathrm{j}_{j k}\right]_{M \times M},
$$

where

$$
\begin{aligned}
& J_{j k}=\frac{\partial F_{j}}{\partial \zeta_{k}}=\frac{1}{\Gamma(1-\beta)} \frac{t_{j}}{M} \sum_{m=1}^{M}\left(t_{j}-\tau_{m}\right)^{-\beta} h_{i}\left(\tau_{m}\right)-\left(a-2 b u_{N}\left(t_{j}\right)\right) p_{i},{ }_{1}\left(t_{j}\right)+2 b \sum_{i=1}^{M} \zeta_{i} p_{i},{ }_{1}\left(t_{j}\right) p_{k},{ }_{1}\left(t_{j}\right) \\
& +c \sum_{i=1}^{M} \zeta_{i} p_{i},{ }_{1}\left(t_{j}\right) \frac{t_{j}}{M} \sum_{m=1}^{M} p_{i},{ }_{1}\left(x_{m}\right)+c p_{i_{1}}\left(t_{j}\right) \frac{t_{j}}{M} \sum_{m=1}^{M} \sum_{i=1}^{M} \zeta_{i} p_{i},{ }_{1}\left(x_{m}\right)
\end{aligned}
$$


The solution of this system gives values of unknown $\zeta_{i}, i=1,2,3, \ldots, M$. The approximate solution at nodal points is calculated by putting $\zeta_{i}, i=1,2,3, \ldots M$ in Eq. (29). Substituting the value of $e_{N},{ }_{M}(t)$ in Eq. (21), we obtain the required solution.

Table 1. Maximum absolute estimated error functions for Beta=1/3 in Test Problem 1

\begin{tabular}{lllcc}
\hline$J$ & $N$ & $M$ & $e_{N},{ }_{M}(t)$ & CPU time (in seconds) \\
\hline 0 & 2 & 4 & $8.91069 \times 10^{-6}$ & 0.02475 \\
1 & 4 & 8 & $5.76824 \times 10^{-8}$ & 0.9087 \\
2 & 8 & 16 & $8.51438 \times 10^{-9}$ & 1.3421 \\
3 & 16 & 32 & $6.16606 \times 10^{-12}$ & 7.5321 \\
4 & 32 & 64 & $1.90067 \times 10^{-13}$ & 19.0956 \\
5 & 64 & 128 & $3.20924 \times 10^{-17}$ & 24.1589 \\
6 & 128 & 256 & $4.21440 \times 10^{-18}$ & 35.2157 \\
7 & 256 & 512 & $3.25710 \times 10^{-19}$ & 53.8651 \\
\hline
\end{tabular}

\section{Numerical applications}

Here we give an example and solve it by the proposed methods discussed above. The notation $e_{N},{ }_{M}(t)$ is used for maximum absolute estimated error functions at $N$ nodal points.

Test Problem 1. Consider a fractional Volterra model for population growth of a species in a closed system [23]

$$
\frac{d^{\beta} u(t)}{d t^{\beta}}=a u(t)-b u^{2}(t)-c u(t) \int_{0}^{t} u(x) d x,
$$

if we take $\beta=1$, then the analytical solution is given by [19]

$$
u(t)=e^{-t}-1+\frac{1}{1+\left(1 / u_{0}-1\right) e^{-t}}
$$

The method is applied to this test problem with parameters as $u(0)=0.1, a=1, b=1, c=1$. The estimated error functions for $\beta=1 / 3$ and $\beta=1 / 2$ of these approximate solutions are shown in Table 1 and Table 2 . In order to obtain accuracy of order $10^{-19}$ for $\beta=1 / 3$ and $10^{-18}$ for $\beta=1 / 2$, we have to take at least $N=256$ numbers of nodal points. From Fig. 1 we see that the accuracy of the technique improves by increasing the number of nodal points. For the solution of this nonlinear system, we applied the Broydens technique. The initial guess for this technique was taken zero and iterations were terminated when the convergent criterion $10^{-19}$ was satisfied.

Table 2. Maximum absolute estimated error functions for Beta=1/2 in Test Problem 1

\begin{tabular}{cllcc}
\hline$J$ & $N=2^{J+I}$ & $M$ & $e_{N},{ }_{M}(t)$ & CPU time (in seconds) \\
\hline 0 & 2 & 4 & $6.15069 \times 10^{-6}$ & 0.01321 \\
1 & 4 & 8 & $3.51619 \times 10^{-8}$ & 0.8214 \\
2 & 8 & 16 & $4.32865 \times 10^{-9}$ & 1.2316 \\
3 & 16 & 32 & $2.14803 \times 10^{-12}$ & 6.0932 \\
4 & 32 & 64 & $6.48502 \times 10^{-14}$ & 17.1572 \\
5 & 64 & 128 & $5.97205 \times 10^{-16}$ & 25.4908 \\
6 & 128 & 256 & $7.57531 \times 10^{-17}$ & 37.0137 \\
7 & 256 & 512 & $3.07869 \times 10^{-18}$ & 53.1702 \\
\hline
\end{tabular}




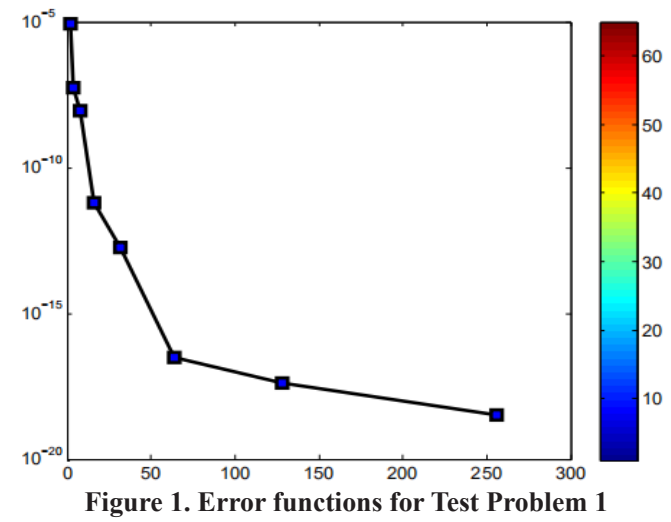

\section{Conclusion}

In this article, the HWCM has been implemented for the FVM for population growth model and we have applied the technique to one example. The results show that the technique is efficient and accurate for different choices of collocation points. The numerical results demonstrate that expanding the parameter $N$ of the proposed technique enhances the exactness of the approximate solution. Furthermore, the residual error functions utilizing distinct numbers of nodal points are also calculated. From the numerical results, it has seen the method gives good results for considered problems.

\section{References}

[1] U. Lepik, H. Lepik. Haar wavelets with applications. Springer. 2014.

[2] L. Debnath. Wavelet transform and their application. Springer. New York: Birkhauser; 2015.

[3] A. Raza, A. Khan. Haar wavelet series solution for solving neutral delay differential equations. J. King Saud Uni. -Sci. 2019; 31(4): 1070-1076.

[4] A. Raza, A. Khan. Approximate solution of higher order two point boundary value problems using uniform haar wavelet collocation method. Springer Proc. in Math. and Stat. 2019; 72: 209-220.

[5] Siraj-ul-Islam, I. Aziz, B. Sarler. The numerical solution of second-order boundary-value problems by collocation method with the Haar wavelets. Math. Comp. Model. 2010; 52: 1577-1590.

[6] M. Caputo. Linear models of dissipation whose q is almost frequency independent. Geophys. J. R. Astr. Soc. 1967; 13: 529-539.

[7] I. Podlubny. Fractional differential equations. Academic press. New York: 1999.

[8] K. Oldham, J. Spanier. The Fractional Calculus. Academic Press. New York: 1974.

[9] U. Lepik. Numerical solution of evolution equations by the Haar wavelet method. Appl. Math. Compt. 2007; 185: 695 $-704$.

[10] R. Bellman, K. L. Cooke. Differential-Difference equations. Academic Press. New York: London; 1963.

[11] A. A. Kilbas, H. M. Srivastava, J. J. Trujillo. Theory and applications of fractional differential equations. NorthHolland Math. Studies. 2006.

[12] A. Bellen, M. Zennaro. Numerical methods for delay differential equations. Clarendon Press. Oxford: Oxford Science Publications; 2003.

[13] K. B. Datta, B. M. Mohan. Orthogonal functions in systems and control. World Scientific. Singapore. 1995.

[14] K. Diethelm, N. J. Ford. Analysis of fractional differential equations. J. Math. Anal. Appl. 2002; 265: 229-248.

[15] S. G. Samko, A. A. Kilbas, O. I. Marichev. Fractional integrals and derivatives: Theory and applications. Yverdon: Gordon and Breach; 1993.

[16] H. Kocak, A. Yildirum. An efficient new iterative method for finding exact solutions of nonlinear time-fractional partial differential equations. Nonlinear Anal.: Model. Control. 2011; 16(4): 403-414.

[17] H. Xu. Analytical approximations for a population growth model with fractional order. Commun Nonlinear Sci Numer. 2009; 14: 1978-1983.

[18] S. Momani, R. Qaralleh. Numerical approximations and pade approximants for a fractional population growth model. Appl. Math. Model. 2007; 31: 1907-1914.

[19] K. G. TeBeest. Numerical and analytical solutions of volterras population model. SIAM Rev. 1997; 39: 484-493.

[20] R. Small. Population growth in a closed model, mathematical modelling: classroom notes in applied mathematics SIAM. Philadelphia. 1997; 39: 484-493. 
[21] I. Aziz, R. Amin. Numerical solution of a class of delay differential and delay partial differential equations via haar wavelet. Appl. Math. Model. 2016; 40: 10286-10299.

[22] Siraj-ul-Islam, I. Aziz, F. Haq. A comparative study of numerical integration based on haar wavelets and hybrid functions. Compu. and Math. with Appl. 2010; 59: 2026-2036.

[23] S. Yuzbasi. A numerical approximation for volterras population growth model with fractional order. Appl. Math. Model. 2013; 37: 3216-3227. 